\title{
Infra-red thermography estimate of deposited heat load dynamics on the lower tungsten divertor of WEST
}

\author{
N. Fedorczak ${ }^{1}$, J. Gaspar ${ }^{2}$, M. Firdaouss ${ }^{1}$, V. Moncada ${ }^{1}$, A. \\ Grosjean $^{1}$, R. Dejarnac ${ }^{3}$, S. Brezinsek ${ }^{4}$, E. Tsitrone ${ }^{1}$, J. \\ Bucalossi $^{1}$, T. Loarer $^{1}$ and the WEST team ${ }^{3}$ \\ ${ }^{1}$ CEA, IRFM, F-13108 Saint-Paul-Lez-Durance, France \\ 2 Aix Marseille Univ, CNRS, IUSTI, Marseille, France \\ ${ }^{3}$ Institute of Plasma Physics, The Czech Academy of Sciences, Za Slovankou 3, 182 \\ 00 Prague 8, Czech Republic \\ ${ }^{4}$ Forschungszentrum Jlich, Institut fr Energie- und Klimaforschung Plasmaphysik, \\ 52425 Jlich, Germany \\ ${ }^{5}$ See http://west.cea.fr/WESTteam \\ E-mail: nicolas.fedorczak@cea.fr
}

\begin{abstract}
A thermography inversion algorithm (TEDDY) was developed for the physical exploitation of WEST infra-red thermography (at $3.9 \mu \mathrm{m})$ of uncooled graphite tiles coated with $12 \mu \mathrm{m}$ of tungsten, located at the lower divertor. The numerical scheme features a conservative finite volume approach with the full temperature non linearity of thermal properties of material composing the tile. The inversion algorithm is validated against synthetic test cases built from standardized software. A first insitu quantification of tungsten surface emissivity and reflected luminance along an outer target coated tile is then detailed. This method relies on a comparison of thermography data with absolute temperature measurements from embedded thermal sensors. Low surface emissivity $(\epsilon \approx 0.05)$ is estimated in the common outer strike point area, while increasing to about 0.1-0.15 far from it, possibly due to deposited impurity layers. The heat flux inversion from corrected thermography temperature is illustrated on an L-mode plasma heated with $4 \mathrm{MW}$ of input power, featuring a strike point sweeping of $30 \mathrm{~mm}$ along the divertor tile. Power balance and sweeping dynamics are consistently recovered by the heat flux inversion, while the radial profile of parallel heat flux remapped to outer midplane features a gaussian-exponential shape common to diverted configurations in low dissipation divertor regime.
\end{abstract}




\section{Introduction}

One of the primary task of the WEST tokamak is to test the actively cooled tungsten monoblock technology that will equip the lower divertor of ITER [1]. In a staged approach, WEST operation has started with an uncooled lower divertor composed of graphite tiles coated with tungsten [2], together with a series of actively cooled tungsten monoblocks covering one toroidal sector of 30 degrees. Heat and particle loads impacting this divertor are characterized with a set of complementary diagnostics: flush mounted Langmuir probes, arrays of embedded thermal sensors (thermocouples [3] and fibre Bragg gratings [4]), and infra red thermography [5]. This contribution focuses on the inversion of surface heat loads from $\lambda_{I R}=3.9 \mu \mathrm{m}$ infra-red thermography of uncooled graphite tiles coated with tungsten, with a spatial resolution of about $3 \mathrm{~mm}$. These coatings consist of a $2-4 \mu \mathrm{m}$ molybdenum inter-layer and $10-13 \mu \mathrm{m}$ of tungsten, both deposited by a PVD CMSII process [2] (surface roughness is in the range of 2-3 $\mu \mathrm{m}$ ). The paper describes the important steps in the thermal inversion process, including the code TEDDY (Thermography Estimate of Deposited heat DYnamics) equivalent to the THEODOR code [6].

\section{Thermal model of TEDDY}

Heat diffusion in the bulk of divertor tiles is generally a 3D problem: heat diffuses from the exposed surface to the bulk of the tile, it diffuses along the poloidal contour of the tile due to the strong poloidal anisotropy of the deposited heat flux, and it can vary in the toroidal direction if the tiles are toroidally beveled or shaped to create mutual shadowing of leading edges (see section 5). For now, we will consider, as in THEODOR [7], that heat will mostly diffuse through the tile along two main directions: the poloidal (toroidal) direction along the profile of deposited heat flux, noted $x$ in the following, and the direction $y$ along the depth of the tile, perpendicular to the previous one (see figure 1).

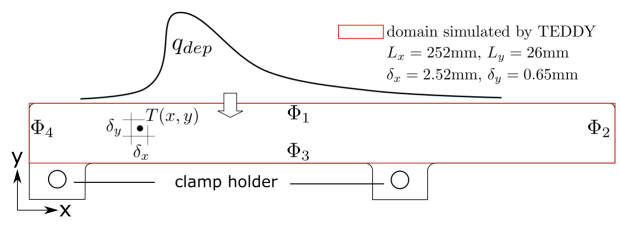

Figure 1. Geometry of the outer strike point graphite tile of WEST lower divertor, showing the $2 \mathrm{D}$ rectangular simulation domain treated by TEDDY. $\left\{\Phi_{k}\right\}$ denotes the boundary surfaces, with $\Phi_{1}$ the surface exposed to the plasma.

Along these two directions, the heat equation reads:

$$
\rho C_{p}(T) \partial_{t} T=-\vec{\nabla} \cdot \vec{q}
$$


where $\rho$ is the volumetric mass in $\mathrm{kg} \cdot \mathrm{m}^{-3}$ of the material composing the tile and $C_{p}$ the specific heat capacity in $\mathrm{J}_{\mathrm{kg}} \mathrm{kg}^{-1} \cdot \mathrm{K}^{-1}$. $T$ denotes the local temperature of the tile in $\mathrm{K}$ and $\vec{q}$ the heat flux in W.m ${ }^{-2}$. In the bulk of the tile, the heat flux along the direction $k$ is described by the Fourier law: $q_{k}=\lambda^{(k)}(T) \partial_{k} T$ where $\lambda^{(k)}$ is the thermal conductivity of the material (in W. $\mathrm{m}^{-1} \cdot \mathrm{K}^{-1}$ ) along the direction $k$. The thermal conductivity is possibly anisotropic as it can be the case for carbon fiber composite [8], although not for graphite. Note also that the thermal properties $C_{p}$ and $\lambda$ are generally strongly varying with material temperature, as shown in figure 2 for graphite. For sake of numerical convenience, the temperature dependance of $\rho C_{p}(T)$ and $\lambda(T)$ are approximated with exponential functions, i.e $\lambda^{(x)}(T)=\lambda_{0}^{(x)}+\lambda_{1}^{(x)} \exp \left(\beta_{\lambda}^{(x)} T\right)$. This description is accurate within a few percents on the range of temperature variations of interest (300 to $2000 \mathrm{~K}$ ).

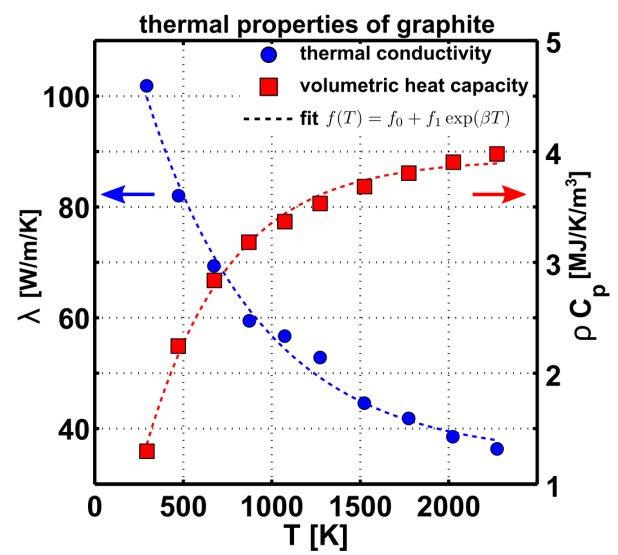

Figure 2. Temperature dependance of thermal properties of graphite.

On the exposed tile surface ( $\Phi_{1}$ in figure 1$)$, the deposited heat flux $q_{\text {dep }}$ has to be considered in the $y$ component of $\vec{q}$. In principle, Planck radiation from boundary surfaces has to be included as well: $q_{\text {rad }}=\epsilon \sigma\left(T_{\text {surf }}^{4}-T_{a m b}^{4}\right)$ where $\epsilon$ is the effective surface emissivity, $\sigma$ the Stefan-Boltzmann constant, $T_{\text {surf }}$ and $T_{a m b}$ the local surface temperature and the ambient temperature, in K. For tungsten surfaces, $\epsilon \leq 0.1$, so that for surface temperatures below $1000 \mathrm{~K}$, the radiated heat flux is below $5 \mathrm{~kW} . \mathrm{m}^{-2}$, far below usual incident heat fluxes. Of course such term has to be considered for the thermalisation of tiles between plasma operation, but will be further neglected in the description of the thermal dynamics of the tile during plasma exposure. Similarly, heat transfer through to back surface $\Phi_{3}$ due to mechanical binding of the tile to support plates will be neglected.

The tile is decomposed on a uniform $(x, y)$ cartesian grid of size $\left(N_{x}, N_{y}\right)$ with unitary elements of size $\left(\delta_{x}, \delta_{y}\right)$ and time is decomposed following a constant time step $\delta_{t}$. Equation 1 is discretized following a first order conservative finite volume scheme. To do so, the divergence of the Fourier heat flux is rewritten as a laplacian of an effective potential as in THEODOR [6]: $\nabla_{k} \cdot\left(\lambda^{(k)} \nabla_{k} T\right) \equiv \nabla_{k}^{2}\left(\Lambda^{(k)}\right)$ where $k$ denotes the direction 
$x$ or $y$ and $\Lambda^{(k)}=T \lambda_{0}^{(k)}+\frac{\lambda_{1}^{(k)}}{\beta_{\lambda}^{(k)}} \exp \left(\beta_{\lambda}^{(k)} T\right)$ using the exponential approximation of $\lambda^{(k)}(T)$. The numerical scheme reads for the $(\mathrm{x}, \mathrm{y})$ pixel $(\mathrm{i}, \mathrm{j})$ :

bulk, $\rho C_{p}\left(T_{i, j}\right) \partial_{t} T_{i, j}=\frac{1}{\delta_{y}^{2}}\left(\Lambda_{i, j-1}^{(y)}+\Lambda_{i, j+1}^{(y)}-2 \Lambda_{i, j}^{(y)}\right)+\frac{1}{\delta_{x}^{2}}\left(\Lambda_{i-1, j}^{(x)}+\Lambda_{i+1, j}^{(x)}-2 \Lambda_{i, j}^{(x)}\right)$

$\Phi_{1}, \quad \rho C_{p}\left(T_{i, N_{y}}\right) \partial_{t} T_{i, N_{y}}=\frac{1}{\delta_{y}^{2}}\left(\Lambda_{i, N_{y}-1}^{(y)}-\Lambda_{i, N_{y}}^{(y)}\right)+\frac{1}{\delta_{y}} q_{d e p, x}+\frac{1}{\delta_{x}^{2}}\left(\Lambda_{i-1, N_{y}}^{(x)}+\Lambda_{i+1, N_{y}}^{(x)}-2 \Lambda_{i, N_{y}}^{(x)}\right)$

$\Phi_{3}, \quad \rho C_{p}\left(T_{i, 1}\right) \partial_{t} T_{i, 1}=\frac{1}{\delta_{y}^{2}}\left(\Lambda_{i, 2}^{(y)}-\Lambda_{i, 1}^{(y)}\right)+\frac{1}{\delta_{x}^{2}}\left(\Lambda_{i-1, N_{y}}^{(x)}+\Lambda_{i+1, N_{y}}^{(x)}-2 \Lambda_{i, N_{y}}^{(x)}\right)$

$\Phi_{2}, \Phi_{4}$, see $\Phi_{3}$.

To simplify, the time evolution of the temperature matrix can be written as

$$
\partial_{t} T=L \Lambda+K q_{d e p}
$$

where $L$ and $K$ are matrices dependent on the local temperature. For sake of generality, we can also assume the existence of a thermal resistance at the surface of the tile [9], to describe effect of surface layers that can be loosely bounded. The thermography surface temperature $T_{\text {surf }}$ would be linked to the top surface of the bulk of the tile $T_{i, N_{y}}$ by $T_{\text {surf }, i}(t)=T_{i, N_{y}}(t)+\alpha_{s} q_{d e p, i}(t)$, with $\alpha_{s}$ the thermal resistance of the surface layer, in $\mathrm{K} . \mathrm{m}^{2} \cdot \mathrm{W}^{-1}$. For graphite tiles of the WEST divertor, the tungsten coating is 12 $\mu \mathrm{m}$ thick. Assuming a perfect thermal bound (strong assumption) with the graphite substrate (through a molybdenum compliance layer), the thermal resistance of such layer is $\alpha_{s}=85 \cdot 10^{-9} \mathrm{~K} . \mathrm{m}^{2} . \mathrm{W}^{-1}$. For typical heat loads in the range of $1 \mathrm{MW} \cdot \mathrm{m}^{-2}$, the difference of temperature between the tile surface and the surface layer is only 0.1 $\mathrm{K}$, thus negligible. For sake of generality, a surface resistance is included in the thermal model, but set to 0 if not otherwise specified.

Forward model $q_{d e p} \rightarrow T$. Given a spatio-temporal evolution of incident heat flux $q_{d e p}$, Equation 2 is solved with a simple forward scheme:

$$
T\left(t+\delta_{t}\right)=T(t)+\delta_{t}\left(L(t) \Lambda(t)+K(t) q_{d e p}(t)\right)
$$

and

$$
T_{\text {surf }}\left(x, t+\delta_{t}\right)=T_{N_{y}}\left(x, t+\delta_{t}\right)+\alpha_{s} q_{\text {dep }}\left(t+\delta_{t}\right)
$$

The time step is chosen small enough to respect the stability criterion of Fourier equations.

Reverse model $T_{\text {surf }} \rightarrow q_{\text {dep }}$. Given the spatio temporal evolution of surface temperature $T_{\text {surf }}$, Equation 2 is solved for the incident heat flux, following a staged scheme. First, the bulk tile temperature is prescribed to be the initial $T_{\text {surf }}$ at the tile surface $\left(j=N_{y}\right)$ and null gradient along $y$. Then for each time step:

- bulk temperature is evolved with heat diffusion only $T^{*}=T(t)+\delta_{t} L(t) \Lambda(t)$

- incident heat flux is calculated from the evolution of surface temperature $q_{\text {dep }}(t)=$ $\frac{T_{s u r f}\left(t+\delta_{t}\right)-T_{j=N_{y}}^{*}}{K(t) \delta_{t}+\alpha_{s}}$ 
- bulk temperature at the next time step is defined as $T\left(t+\delta_{t}\right)=T^{*}$ away from the exposed surface, and incremented by the incident heat flux at the exposed surface: $T_{j=N_{y}}\left(t+\delta_{t}\right)=T_{j=N_{y}}^{*}+K(t) \delta_{t} q_{d e p}(t)$

\section{Validation of TEDDY on synthetic test cases}

A series of synthetic test cases are considered for validating the heat flux inversion done with TEDDY. First, a heat flux pattern is simulated at the surface of the tile with the PFCflux code [10]: parallel heat flux is projected from the midplane of a WEST magnetic equilibrium, assuming a decaying exponential profile across flux surfaces (of width or decay length $\lambda_{q}^{u}$ ). The projection to the tile surface takes into account magnetic shadowing by the tokamak wall elements, in particular mutual tile shadowing and divertor shadowing by the divertor baffle. At the target, an additional radial spreading (gaussian smoothing of width $S$ ) is included to account for radial diffusion of the streaming plasma along the magnetic divertor leg. Then, the tile temperature is evolved during temporal heat flux pulses of a few seconds using 3D simulations (3D realistic tile geometry with toroidal shadowing) using the ANSYS software. The resulting surface temperature evolution is finally extracted close to the trailing edge of the tile (away from the toroidal shadowing), and inverted with TEDDY.
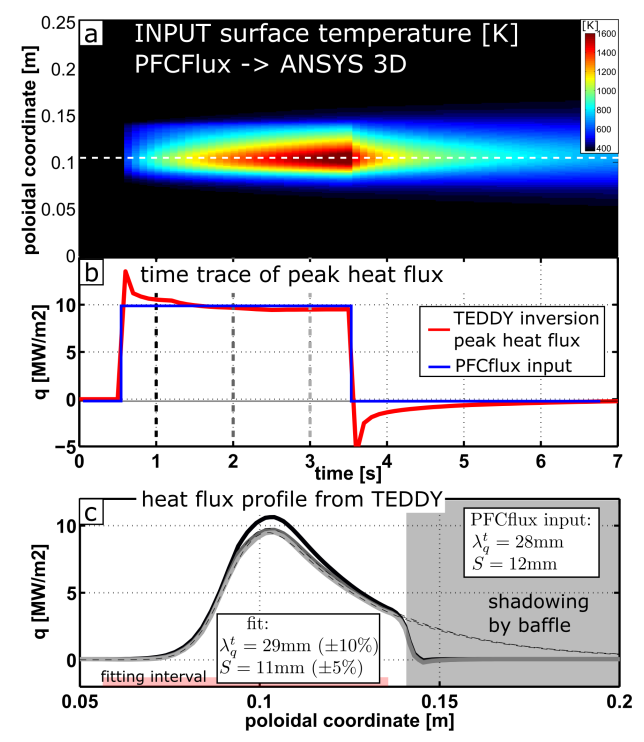

Figure 3. a) Input surface temperature calculated with PFCFlux-ANSYS3D. b) time trace of peak heat flux inverted from TEDDY (red) compared to PFCFlux input (blue). c) poloidal profile of heat flux inverted from TEDDY, at three times (dashed lines in graph b). The inversion recovers the input width parameters $(S$ and $\lambda)$ with less than $10 \%$ error.

Results for one validation case are shown in figure 3. The shape of the heat flux pattern given by TEDDY are nicely fitted with the common $S-\lambda_{q}$ function [11] giving width parameters ( $S$ and $\lambda$ along the target) in very close agreement with the input set 
by PFCflux (4\% error for $\lambda_{q}$ and $10 \%$ error for $S$ ). Additionally, the inversion recovers the magnetic shadowing produced by the divertor baffle. The absolute amplitude of the peak heat flux (10 MW.m ${ }^{-2}$ ) also matches the input, as well as the global temporal evolution. However, the inversion produces overshoots at the two sharp fronts of the temporal heat pulse. At the front up, the overshoot of peak heat flux exceeds the reference value by $30 \%$ but converges exponentially towards it with a time scale of about $200 \mathrm{~ms}$. At the front down, the negative overshoot has a similar dynamics but toward negative values. The same observations are obtained for a test case with 2 MW. $\mathrm{m}^{-2}$ instead of $10 \mathrm{MW} \cdot \mathrm{m}^{-2}$. A refinement of discretion parameters of the inversion $\left(\delta_{x}, \delta_{y}, \delta_{t}\right)$ did not show any improvement of the relative amplitude and time scale of these overshoots. They are successfully eliminated by setting an adjusted value of the surface resistance $\alpha_{s}$, as also studied with THEODOR for fast transients [9]. However, such a correction is unphysical in this validation process. It rather results from a different thermal modeling between TEDDY-2D and ANSYS-3D. In particular TEDDY neglects the toroidal diffusion of heat toward the shadowed fraction of the tile, which probably leads to an artificial compensation by a stronger heat flux variation during transient phases.

\section{Effect of toroidal shadowing on $2 \mathrm{D}$ thermography inversion}

The coated graphite tiles of the WEST lower divertor are $30 \mathrm{~mm}$ wide in the toroidal direction (same as tungsten monoblocks) and shaped with a toroidal bevel of $1^{\circ}$. In standard magnetic configurations, the toroidal shadow around strike point is in the range of $20-40 \%$ of the tile width, meaning that more than $50 \%$ of the toroidal contour of the tile is exposed to a uniform parallel heat flux, as illustrated in figure 4 . In these conditions the 3D heat inversion problem is approximated as follow: heat diffusion is solved dynamically with TEDDY in 2D along the tile depth and and the poloidal direction, and effect of toroidal heat diffusion from exposed to shadowed tile area is simply taken into account by a correction factor for the incident heat flux that depends on the shadowed fraction. To evaluate this correction factor, TEDDY is applied to the toroidal/depth section of the tile as shown in figure 4. A set of synthetic cases are built (scan of shadowing fraction and indicent heat flux) featuring a uniform heat flux on the exposed fraction of the tile, and zero flux on the shadowed area (neglecting radiation fluxes). The forward mode of TEDDY returns the time evolution of the surface temperature along the toroidal direction. Temperature is then averaged on a region of interest around the toroidal center of the tile (see figure 5) and TEDDY is run in the inverse mode assuming that the heat flux is now uniform on the surface of the tile. The time dynamics of the resulting effective heat flux shows indeed transient behaviors at the beginning or end of the incident heat pulse. Overshoots appear for small shadowing fractions $(f<0.5)$ to compensate a thermal flux toward the shadowed region. On the other hand smooth dynamics appear for large shadowing fractions $(f>0.5)$, because the region of interest is now in the shadowed part of the tile, thus beeing heated by 
the thermal transert from the exposed area. After a transient time, the effective heat flux reaches a constant value $q_{\text {eff }}$ which is then compared to the input one $q_{\perp}$, and the ratio defines the correction factor shown in figure 4 . This correction factor should thereofre be considered as a geometrical correction in the steady-state limit, but cannot be Overall, these synthetic results are well organized around a unique trend, that is fitted with an hyperbolic tangent. This simple analytic approximation will serve in the following to estimate a correction factor function of the shadowed fraction of the tile. Given the range of usual shadowing in the WEST experiments, a correction of about $10-30 \%$ of the deposited heat flux value is expected. This correction will be applied to thermography inversion of WEST plasma experiments taking into account the local shadowing fraction calculated from the 3D magnetic geometry.

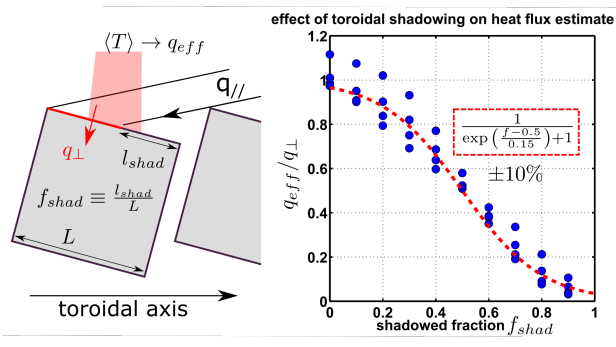

Figure 4. left: idealized geometry of tiles in toroidal/vertical plan used for assessing the effect of toroidal shadowing with TEDDY. The red line of sight defines the region at the tile surface where thermography temperature is averaged. Right: variation of the heat flux correction factor function of shadowing fraction as calculated using TEDDY. The dashed curve represent an analytical approximation.

\section{Preliminary in-situ estimate of surface temperature of tungsten coated graphite tiles from infra-red thermography}

In WEST, the temperature dynamics of the surface of divertor tiles is provided by a large set of infra-red thermography systems [5], designed with the primary purpose of wall protection. Analysis presented in this work are based on infra-red data at $3.9 \mu \mathrm{m} \pm 0.1 \mu \mathrm{m}$ collected by an actively cooled optical endoscope located at the top of the chamber looking down to the lower divertor with a resolution of $2.8 \mathrm{~mm}$ per pixel. The thermography data is absolutely calibrated and can be translated into apparent black body temperature of the wall elements. As illustrated in figure 5, a tile of interest is selected at the outer strike point region, at a toroidal position where the ripple modulation of the incidence angle of magnetic field lines provides the maximum deposited heat flux. A poloidal profile of apparent black body temperature is extracted at the toroidal center of the tile to avoid spurious infra-red light emitted from cavities between tiles.

On tungsten surfaces, an emissivity $\epsilon<1$ has to be accounted for to transform the apparent black body temperature into surface temperature. Moreover, it is also 


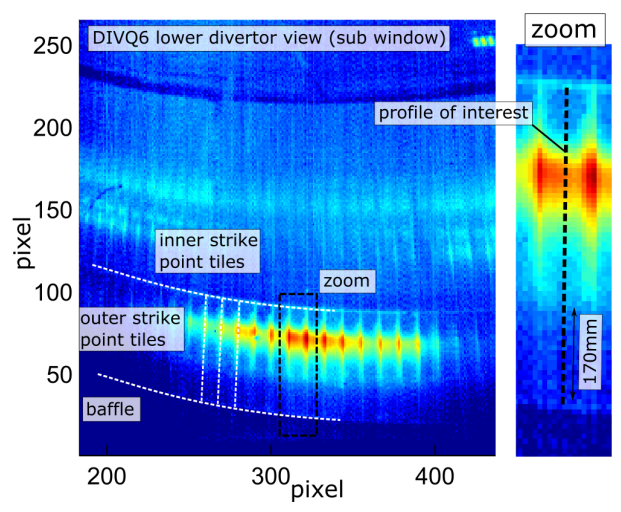

Figure 5. illustration of the thermography data (apparent black body temperature) from a lower divertor endoscope. A poloidal profile is defined along a tile of interest.

important to consider infra-red reflections on the surface of interest from emissions coming from the rest of the chamber. This is particularly important in a metallic environment of high reflectivity. The reflected luminance has two main contributions: constant ambient luminance from the thermalized $\left(T_{a m b}=90^{\circ} \mathrm{C}\right)$ stain-less steel wall panels covering most of the first wall, and reflections of dynamic and localized hot surfaces during plasma operation, including the self reflection of the lower divertor luminance. The second contribution is the most complex to handle, and requires 3D photonic transport simulations [12] currently in preparation for WEST. In the remaining of this work it will be simply neglected, considering that the lower divertor is the most exposed component during plasma operation and assuming that self reflection on the chamber components induces a weak luminance contribution to the lower divertor thermography. Reflection from wall ambiance is however kept, considering the large area of emission and the stationarity of this contribution. The simplest relation between apparent black body temperature $\left(T_{I R}\right)$ and surface temperature $T_{\text {surf }}$ can be written using the Planck luminance function $L_{p}(T)$ :

$$
L_{p}\left(T_{I R}\right)=\epsilon L_{p}\left(T_{\text {surf }}\right)+(1-\epsilon) \epsilon_{a m b} L_{p}\left(T_{a m b}\right)
$$

The reflected luminance is simply modeled as the Planck emission of the wall ambiance (wall at temperature $T_{a m b} \approx 90^{\circ}$ ), weighted by an effective emissivity $\epsilon_{a m b}$. This parametrization provides an approximation of multiple reflections on stainless-steel panels ( diffuse and specular) and shadowing effects from local objects like baffle. The problem of estimating surface emissivity and contribution from the simplified ambiance reflections translates into the estimate of surface emissivity $\epsilon$ and effective ambiance emissivity $\epsilon_{a m b}$ along the profile of the tile. This can simply be obtained from at least two set of $\left(T_{I R}, T_{\text {surf }}, T_{a m b}\right)$ data. The true surface temperature can be estimated from thermocouples or fiber Bragg gratings embedded a few millimeters from the surface of equivalent tiles also located close to the maximum of toroidal ripple.

A few tens of minutes after a standard pulse, temperature becomes uniform in the tile so that these embedded measurements do provide a reliable estimate of the 


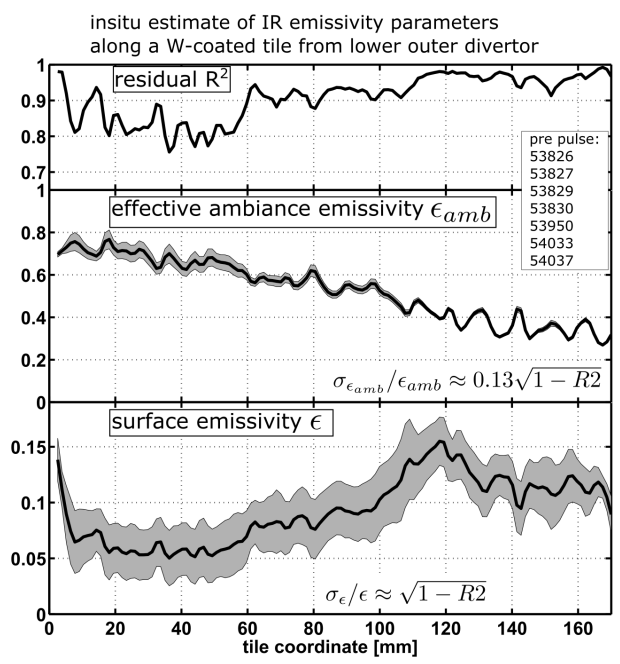

Figure 6. Profiles of surface emissivity and effective ambiance emissivity derived from the comparison between infra-red thermography and embedded thermal measurements

surface temperature. A set of seven post pulse phases have been selected during the last experimental campaign, providing an increment of the tile temperature from $T_{\text {surf }}=90^{\circ}$ to about $200^{\circ}$, while maintaining the actively cooled wall at $90^{\circ}$. A least square minimization over these set of data allows to extract the optimal values of $\epsilon$ and $\epsilon_{a m b}$ along the tile together with a minimization residual $R^{2}$. In order to estimate an amplitude of dispersion of $\epsilon$ and $\epsilon_{a m b}$ from the amplitude of the residual, a preliminary sensitivity analysis was performed. It consists in a Monte Carlo sampling of synthetic experimental data, assuming uncertainty on both surface emissivity and infrared luminance calibration at low temperature. The most conservative relations between residual and dispersion levels are found to be $\sigma_{\epsilon} / \epsilon \approx 1.0 \sqrt{1-R^{2}}$ and $\sigma_{\epsilon_{a m b}} / \epsilon_{\text {amb }} \approx$ $0.13 \sqrt{1-R^{2}}$. Results are summarized in figure 6 . The effective ambiance emissivity reaches about 0.8 on the inner boundary of the tile, suggesting that the reflected ambient luminance results from multiple reflections on stainless-steel wall panels (surface emissivity normally in the range of 0.3 ). The apparent decrease of the effective ambiance emissivity along the tile profile can be potentially explained by the gradual shadowing of the thermalized ambiance from the divertor baffle.

Strikingly, the surface emissivity of the tungsten coated tile exhibits quite low values down to $\epsilon \approx 0.05$ on the tile area where the magnetic strike point commonly lies $(s \in[20,60] \mathrm{mm})$. Away from this area emissivity increases up to 0.1-0.15, where visual inspection suggests surfaces polluted by deposited mater. Overall the regression is satisfactory over the profile, but shows relatively weak residuals $\left(R^{2} \approx 0.8\right)$ in the region of plasma exposure, interpreted as an uncertainty of $\approx 45 \%$ of the value of surface emissivity. This can suggest a possible evolution of the state of the tile surface across the selected range of pulses $(<200)$, which will be investigated through an extended analysis of surface emissivity variation through the entire campaign. Note finally that this 

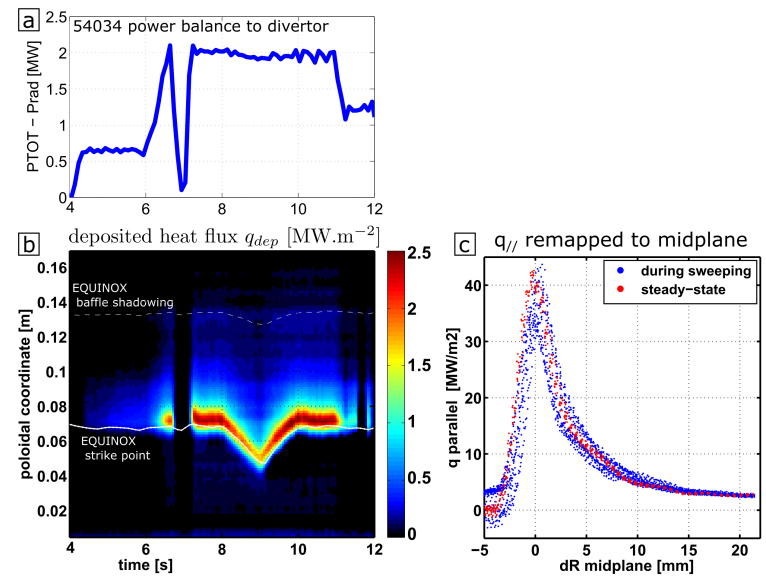

Figure 7. TEDDY inversion from WEST 54034 pulse. a) time trace of total input power minus total radiated power. b) spatio temporal evolution of the heat flux absorbed by the tile surface. The magnetic strike point position calculated by EQUINOX is shown, as wel as the frontier of the magnetic shadowing by the baffle. The strike point is swept over $30 \mathrm{~mm}$ in between $8 \mathrm{~s}$ and $10 \mathrm{~s}$. c) profiles of parallel heat flux remapped to outer midplane during sweeping and in the steady-state phase $(\mathrm{t}=10.5 \mathrm{~s})$, showing relatively small differences.

estimate of surface emissivity could be affected by an error on the absolute calibration of the optical system: it should rather be recalled effective emissivity.

\section{Application of TEDDY to WEST experiments}

The effective surface emissivity of the tile and the reflected ambiance emission have been estimated, but reflections from other hot surfaces and self-reflections form the divertor are currently neglected. With that in mind, the surface temperature can be deduced from the infra-red apparent black body temperature using equation3. A Monte Carlo sensitivity analysis was performed with TEDDY on synthetic cases to translate the uncertainty of surface emissivity into uncertainty of heat flux amplitude, resulting in $\frac{\sigma_{q}}{q} \approx 0.25 \frac{\sigma_{\epsilon}}{\epsilon}$. With an uncertainty of $\epsilon$ in the range of $50 \%$, this translates in an uncertainty of the incident heat flux in the range of about $13 \%$.

An inversion of the incident heat flux with TEDDY is illustrated in figure 7, featuring a high power phase in L-mode with a sweeping of $30 \mathrm{~mm}$ of the magnetic strike point during the heated phase. The heat flux patterns follows nicely the wave form the power balance as well as strike point motion, while the deposited heat flux amplitude slightly decreases as the strike point moves inward. This is due to an increase of the magnetic flux expansion during this inward motion, lowering the magnetic field incidence angle. When the deposited heat flux is projected along the parallel direction $q_{\|}=q_{\text {dep }} / \sin \alpha$, and then remapped to the outer-midplane following the magnetic field lines, the resulting parallel heat flux profile is found to be relatively constant during the strike point sweeping (figure $7 \mathrm{c})$. 


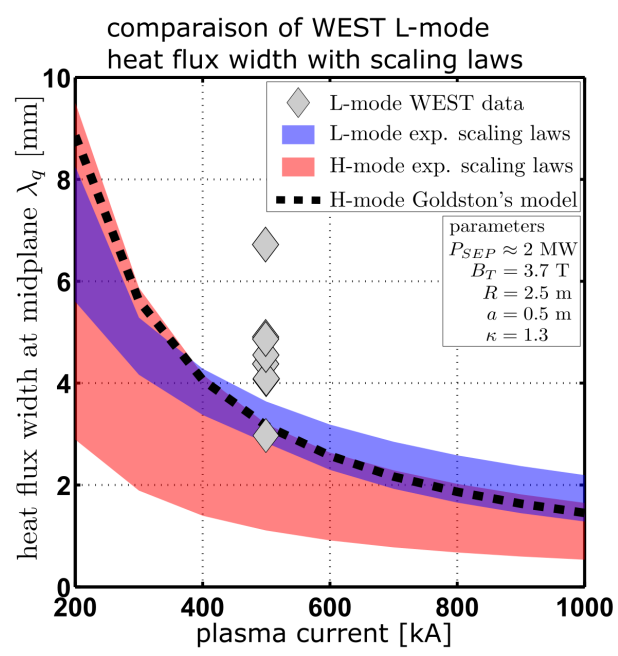

Figure 8. Heat flux width measured in WEST L-mode discharges at $I_{P}=500 \mathrm{kA}$ with predictions from multi-machine scaling built from L-mode and $\mathrm{H}$-mode conditions. Is also shown predictions from the drift model of Goldston.

Note that this parallel heat flux profile follows the common Gaussian-exponential shape found in diverted geometry: a fit gives $S^{t}=3.8 \mathrm{~mm}$ and $\lambda_{q}^{t}=9 \mathrm{~mm}$ at the target, and $\lambda_{q}=3 \mathrm{~mm}$ at the outer-midplane. The spreading factor $S^{t}$ is quite close to the spatial resolution of the camera $(2.8 \mathrm{~mm})$ and thus probably also include optical broadening effects. Although the ratio of $S^{t} / \lambda_{q}^{t} \leq 0.4$ is common to infra-red measurements in low dissipation divertor regimes [11], it motivates detailed investigations with the very high resolution infra red thermography system installed in WEST [5], currently being analyzed.

Parametric sensitivity of the heat flux width in WEST is under analysis. Most of experiments have been operated at a plasma current of $500 \mathrm{kA}$ and magnetic field of 3.7T, in L-mode confinement regimes. In these specific conditions, the heat flux width $\lambda_{q}$ as measured by infra-red thermography is found to span around $5 \mathrm{~mm}$ with a large variation from about $3 \mathrm{~mm}$ to almost $7 \mathrm{~mm}$, as shown in figure 8 . No clear correlation with a discharge parameter was found. For comparison, figure 8 also illustrates the predictions for WEST made from 2 sets of experimental scaling laws and from a model applicable to H-mode. The L-mode experimental scaling [13] applied to WEST conditions predict a $\lambda_{q}$ value in the range of $3 \mathrm{~mm}$ to $3.5 \mathrm{~mm}$. The H-mode experimental scaling [11] predict a value in the range of $1 \mathrm{~mm}$ to $3 \mathrm{~mm}$ whereas the drift model [14] predicts $3 \mathrm{~mm}$. The parametric range of WEST conditions have been extended and data are beeing analysed. Results will be valuable to consolidate these experimental scaling and validate related models, in particular regarding the impact of total magnetic field and aspect ratio. 


\section{Conclusion}

The thermography inversion algorithm TEDDY is applied to infra-red thermography of uncooled tungsten coated graphite tiles of the current lower divertor of WEST. The numerical algorithm was validated against synthetic test cases built from PFCFlux and ANSYS. Some overshoot artifacts were identified during abrupt transients, featuring time scale in the range of $200 \mathrm{~ms}$ and amplitude excess in the range of $30 \%$ of the reference heat flux, probably due to the neglect of toroidal heat diffusion. Application of the algorithm to infra-red thermography has required a careful estimate of true surface temperature by an assessment of an effective tungsten surface emissivity and reflections, thanks to a comparison with embedded thermal sensors. A preliminary analysis of the WEST tiles suggests that effective tungsten emissivity at $\lambda_{I R}=3.9 \mu \mathrm{m}$ reaches quite low values of $\epsilon \approx 0.05$ over the common outer strike point area. The relatively large uncertainty $(\approx 50 \%)$ associated with this estimate suggests that the surface state could evolve over less than 200 plasma discharges. A more extensive analysis over the whole experimental campaign is foreseen to consolidate this first results. Finally, the thermal inversion from thermography corrected surface temperature shows encouraging results from L-mode heated experiments. Heat load patterns recovers power modulation and strike point sweeping with the accurate dynamics. Ongoing efforts are made to compare the amplitude and shapes of these thermography heat fluxes with independent diagnostics: high resolution infra red-thermography on actively cooled tungsten monoblocks, arrays of Langmuir probes and embedded thermal sensors. Preliminary comparisons suggest a quantitative match of estimate of the peak heat flux from these diagnostics [15].

\section{Acknowledgment}

Work performed under EUROfusion WP PFC. This work has been carried out within the framework of the EUROfusion Consortium and has received funding from the Euratom research and training programme 2014-2018 and 2019-2020 under grant agreement No 633053. The views and opinions expressed herein do not necessarily reflect those of the European Commission.

[1] J. Bucalossi et al. Fusion Engineering and Design, 89(7):907 - 912, 2014. Proceedings of the 11th International Symposium on Fusion Nuclear Technology-11 (ISFNT-11) Barcelona, Spain, 15-20 September, 2013.

[2] M. Firdaouss, C. Desgranges, C. Hernandez, M. Richou, H. Greuner, B. Bswirth, I. ZacharieAubrun, T. Blay, J. Bucalossi, M. Missirlian, F. Samaille, and E. Tsitrone. Fusion Engineering and Design, 124:207 - 210, 2017. Proceedings of the 29th Symposium on Fusion Technology (SOFT-29) Prague, Czech Republic, September 5-9, 2016.

[3] J. Gaspar, Y. Corre, M. Firdaouss, J-L. Gardarein, D. Guilhem, M. Houry, C. Le Niliot, M. Missirlian, C. Pocheau, and F. Rigollet. Journal of Physics: Conference Series, 745:032091, sep 2016.

[4] J. Gaspar, Y. Corre, J-L. Gardarein, M. Firdaouss, D. Guilhem, M. Houry, G. Laffont, C. Le Niliot, M. Missirlian, C. Pocheau, and F. Rigollet. Nuclear Materials and Energy, 12:1077 - 
1081, 2017. Proceedings of the 22nd International Conference on Plasma Surface Interactions 2016, 22nd PSI.

[5] X. Courtois, M.H. Aumeunier, C. Balorin, K. Blanckaert, M. Houry, M. Jouve, P. Moreau, C. Pocheau, and H. Roche. Fusion Engineering and Design, 136:1499 - 1504, 2018. Special Issue: Proceedings of the 13th International Symposium on Fusion Nuclear Technology (ISFNT$13)$.

[6] A Herrmann, W Junker, K Gunther, S Bosch, M Kaufmann, J Neuhauser, G Pautasso, Th Richter, and R Schneider. Plasma Physics and Controlled Fusion, 37(1):17-29, jan 1995.

[7] B. Sieglin, M. Faitsch, A. Herrmann, B. Brucker, T. Eich, L. Kammerloher, and S. Martinov. Review of Scientific Instruments, 86(11):113502, 2015.

[8] J. Gaspar, J.-L. Gardarein, F. Rigollet, C. Le Niliot, Y. Corre, and S. Devaux. International Journal of Thermal Sciences, 72:82 - 91, 2013.

[9] A. Herrmann and ASDEX Upgrade team. ECA, 25A:2109-2112, 2001.

[10] M. Firdaouss, T. Batal, J. Bucalossi, P. Languille, E. Nardon, and M. Richou. Fusion Engineering and Design, 98-99:1294 - 1298, 2015. Proceedings of the 28th Symposium On Fusion Technology (SOFT-28).

[11] T. Eich, A.W. Leonard, R.A. Pitts, W. Fundamenski, R.J. Goldston, T.K. Gray, A. Herrmann, A. Kirk, A. Kallenbach, O. Kardaun, A.S. Kukushkin, B. LaBombard, R. Maingi, M.A. Makowski, A. Scarabosio, B. Sieglin, J. Terry, A. Thornton, ASDEX Upgrade Team, and JET EFDA Contributors. Nuclear Fusion, 53(9):093031, 2013.

[12] M.-H. Aumeunier, M. Koan, R. Reichle, and E. Gauthier. Nuclear Materials and Energy, 12:1265 - 1269, 2017. Proceedings of the 22nd International Conference on Plasma Surface Interactions 2016, 22nd PSI.

[13] A. Scarabosio, T. Eich, A. Herrmann, and B. Sieglin. Journal of Nuclear Materials, 438:S426 S430, 2013.

[14] R.J. Goldston. Nuclear Fusion, 52(1):013009, dec 2011.

[15] A. Grosjean et al. in preparation. 